\title{
A Low Complexity Iterative SLM-OFDM for SHF band applications
}

\author{
Sukanya.A.Kulkarni ${ }^{1}$, B.K.Mishra ${ }^{2}$ \\ ${ }^{1}$ Electronics \& Telecommunication Department,Sardar Patel Institute of Technology, \\ Mumbai University,Mumbai,India \\ sukanya_kulkarni@spit.ac.in \\ ${ }^{2}$ Electronics \& Telecommunication Department,Thakur College of Engineering and Technology,Mumbai \\ University,Mumbai,India
}

\begin{abstract}
The transmitted Orthogonal Frequency Division Multiplexing signal in systems such as Wireless LAN, Mobile WiMAX, Digital Video Broadcasting (DVB) operating in the Super High Frequency (SHF) band suffers from high Peak to Average Power Ratio. This is reduced in Selective Level Mapping (SLM)approach but at the expense of increased complexity. In this paper, we propose iterative selective level mapping based on an orthogonal phase sequence set for PAPR reduction with reduced computation complexity. The Golay Complementary pair is used as basis to generate the phase sequence set. Analysis of the aperiodic properties of individual phase sequence and the entire phase sequence set is carried out. It is suggested that mean-square value of auto correlation be an additional measure to choose phase sequence set. Simulations are carried out to assess the PAPR and complexity reduction achieved. The proposed phase sequence set achieves a 1.2 dB and 2.4 dB PAPR improvement over conventional SLM and OFDM respectively for maximum of 16 candidate signals at iterative threshold of $6 \mathrm{~dB}$. The computational complexity reduction achieved is $59 \%$.
\end{abstract}

Index Terms - Complexity, Iterative Selective Level Mapping (ISLM), OFDM, Peak-to-Average Power Ratio (PAPR), Phase Sequence set, SHF

\section{INTRODUCTION}

Orthogonal Frequency division multiplexing $(\mathrm{OFDM})$ is reliable technique for wireless high-rate data transmission over frequency selective fading channels in SHF band.It finds application in physical layer implementationof wireless standards such as Digital Audio Broadcasting (DAB), DigitalVideo Broadcasting (DVB), WLAN standards (802.11), WiMAX (802.16) and Ultra Wide Band (UWB) Communication. One of the major problems of the OFDM system is that it has higher peak to-average-power ratio (PAPR). 
The high peak to average power ratio (PAPR) has a severe impact on power amplifiers forcing it to operate in nonlinear region,which causes inter modulation and out of band radiation. In order to overcome this problem, the power amplifier must operate within its linear region, which implies low efficiency and large power dissipation, which is a disadvantage in wireless applications.

Hence, Selective level mapping [1], a distortion less multiple signal representation method to reduce the PAPR of an OFDM symbol was proposed. In this method,primarily U statistically independent sequences referred to as candidate signals which are phase rotated versions of the original OFDM symbol are generated and sequence with least PAPR is selected for transmission over the channel.

For U candidate signals, computational complexity of conventional SLM increases because of U-1 additional Inverse Fast Fourier Transforms(IFFTs) in SLM compared to OFDM. In literature, methods to reduce complexity based on number of IFFT operations or by having a variable number of candidate signals are found.

In Merged SLM scheme [2] a two stage IFFT is discussed to reduce complexity. Condex matrix based phase sequences [3] has achieved a 59\% reduction in the computational overhead. To reduce the complexity of the conventional SLM scheme, Wang [4] developed two low complexity methods based on conversion matrices to replace additional IFFTblocks but at expense of PAPR degradation. J. Ji,G.Ren and H. Zhang [5] have achieved low computational complexity by designing separate phase rotation vectors for data and pilotsymbols but PAPR performance has worsened. There are various other low complexity schemes proposed in literature [6]- [9] to reduce complexity of conventional SLM based on modifications to IFFT stage. In [10], [11], expectation of PAPR for SLM and low complexity SLM is discussed.

In successive SLM method [11], the candidate signal generation stops as soon as a preset PAPR threshold is reached, reducing the computational complexity. In the SSLM method random phase sequences are used which require side information to be transmitted.

To avoid transmission of side information, orthogonal phase sequence sets have been proposed in [12] to generate U candidate signals in SLM. Further, it is suggested that the component wise product of any two phase sequences (rows) of a phase sequence set are periodic, then there is no improvement in PAPR reduction. Sequences such as Sylvester Hadamard matrices, cyclic Hadamard matrices constructed from m-sequences, Rudin Shapiro sequences and Riemann sequences [13]- [15] exist in literature. Sylvester Hadamard matrices whose rows are Walsh functions are referred to as Walsh-Hadamard matrices in our paper.

This motivates us to propose, low complexity SLM for orthogonal phases sequence sets which is an extension based on the design parameters and approach of [11] referred to as Iterative SLM (ISLM). Brazilian Microwave and Optoelectronics Society-SBMO received 08 Dec 2016; for review 09 Dec 2016 ; accepted 02 Jun 2017 
The phase sequence set for ISLM is constructed based on Golay complementary pairs [17]- [19] referred to as Golay-Hadamard (GH) phase sequence set and we verify the periodicity of the phase sequences of the set. Further, additional criteria of mean square value of autocorrelation of the proposed phase sequence set [16] is evaluated to determine its suitability for ISLM. We analyze the PAPR performance and complexity reduction achieved in low complexity SLM. Further, we compare the improvement in PAPR performance and reduction in complexity of the new phase sequence set and Walsh-Hadamard phase sequence set.

In section II,we discuss Iterative SLM. In section III, recursive construction of Golay-Hadamard phase sequence sets and analysis is presented. The achieved PAPR and complexity reduction analysis are presented and analyzed in section IV. Finally our concluding remarks are provided in section V.

\section{II.ITERATIVE SLM}

In our proposed work, if the PAPR of the OFDM symbol exceeds a certain threshold value, iterative SLM is applied to the data symbol and tested until the PAPR falls below the threshold. The threshold above which SLM is applied is referred to as iterative threshold $\left(\gamma_{\mathrm{th}}\right)$. The maximum number of candidate signals generated to not exceed half the number of subcarriers. The number of assessed candidate signals (U) would be not fixed like in Conventional SLM. The iterative SLM implementation steps in brief are as follows.

The input data stream isencoded using half rate Convolutional Encoder and 16-QAM constellation mapping is performed. The output of constellation mapper referred to as input symbol sequence is thediscrete complex valued sequence expressed as a vector of block length $\mathrm{N}$,

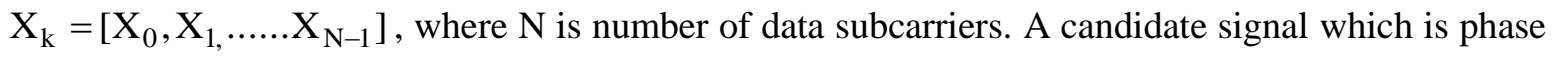
rotated vector of input symbol sequence is generated by element wise multiplication of the input symbol sequenceand phase sequence represented as $\phi_{k}{ }^{(u)}$, both of length $\mathrm{N}$ and $\mathrm{u} €[1, \mathrm{U}-1]$.

$$
X_{k}^{(u)}=X_{k} e^{j \phi_{k}^{(u)}}
$$

Then the time domain OFDM signal generated for each data block given by

$$
\mathrm{x}_{\mathrm{n}}^{(\mathrm{u})}=\frac{1}{\sqrt{\mathrm{N}}} \sum_{\mathrm{k}=0}^{\mathrm{N}-1} \mathrm{X}_{\mathrm{k}}^{(\mathrm{u})} \mathrm{e}^{\mathrm{j} 2 \pi \mathrm{kt} / \mathrm{T}}
$$

where $\mathrm{N}$ denotes number of subcarriers and the IFFT/FFTsize. The Peak-to-Average Power Ratio ( PAPR) of each candidate signal is obtained as 


$$
\operatorname{PAPR}\left\{\mathrm{x}^{(\mathrm{u})}\right\}=\frac{\mathrm{P}_{\text {peak }}}{\mathrm{P}_{\text {avg }}}=10 \log _{10} \frac{\max _{0 \leq \mathrm{n} \leq \mathrm{N}-1}\left|\mathrm{x}_{\mathrm{n}}^{(\mathrm{u})}\right|^{2}}{\mathrm{E}\left[\left|\mathrm{x}_{\mathrm{n}}^{(\mathrm{u})}\right|^{2}\right]}
$$

where $\mathrm{x}^{(\mathrm{u})}=\left[\begin{array}{lllllll}\mathrm{x}_{0}^{(\mathrm{u})} & \mathrm{x}_{1}^{(\mathrm{u})} & \mathrm{x}_{2}^{(\mathrm{u})} & \ldots & \ldots & \mathrm{x}_{\mathrm{N}-1}^{(\mathrm{u})}\end{array}\right], \quad \mathrm{u}=0,1,2 \ldots \mathrm{U}, \mathrm{P}_{\text {peak }}$ represents peak output power, $\mathrm{P}_{\mathrm{avg}}$ means average output power, E [·] denotes the expected value. The above steps are performed recursively until candidate signal with least PAPR for a given iterative threshold is obtained or the maximum number of iterations are reached. The maximum possible iterations are limited to the number of phase sequences (rows) of phase sequence set under consideration. It is also possible that even after exhausting all possible combinations of phase rotated sequences the PAPR is still larger than the threshold value. Complementary Cumulative Distribution function (CCDF) which is a characterization constant related to probability is used as the measurement index to represent the distribution of PAPR. When U mutually independent candidate signals are considered for ISLM, CCDF of PAPR is expressed as follows.

$$
\mathrm{P}_{\mathrm{r}}\left(\operatorname{PAPR}_{\mathrm{SLM}}>\gamma_{\mathrm{th}}\right)=\left(\mathrm{P}_{\mathrm{r}}\left(\operatorname{PAPR}\left\{\mathrm{X}^{(\mathrm{u})}\right\}>\gamma_{\mathrm{th}}\right)\right)^{\mathrm{U}}=\left(1-\left(1-\mathrm{e}^{-\gamma_{\mathrm{th}}}\right)^{\mathrm{N}}\right)^{\mathrm{U}}
$$

where $\gamma_{\text {th }}$ is PAPR threshold.

\section{A. Computational Complexity Analysis}

Computational resources imply the number of complex additions and complex multiplications necessary for a IFFT operation to generate the OFDM signal. Conventional SLM for each candidate signal mapping needs $\left.\left[\mathrm{U}^{*}(1+\mathrm{N} / 2) \log 2(\mathrm{~N})\right)\right]$ complex multiplications and $\left[\mathrm{U}^{*}(\mathrm{~N}) \log 2(\mathrm{~N})\right]$ complex additions for each IFFT. Further U mappings are performed irrespective of whether PAPR may already be reduced below a certain threshold in first few mappings or not. Hence increase in computational complexity increases as U increases.

In ISLM the candidate signal generations stop as soon as PAPR less than threshold value is found. Hence the number of candidate signal mappings generated per OFDM sample may be lesser or at most equal to $\mathrm{U}$. In such cases, the average number of assessed candidate signals, $\mathrm{E}$ [ $\left.\mathrm{U}_{\mathrm{ISLM}}\right]$ is a suitable measure. Mathematically, E [ $\mathrm{U}_{\mathrm{ISLM}}$ ] is given by [11],

$$
\mathrm{E}\left[\mathrm{U}_{\text {ISLM }}\right]=\frac{1-\left(1-\left(1-\mathrm{e}^{-\gamma_{\mathrm{th}}}\right)^{\mathrm{N}}\right)^{\mathrm{U}}}{1-\left(1-\mathrm{e}^{-\gamma_{\mathrm{th}}}\right)^{\mathrm{N}}}
$$

and it would be less than that of conventional SLM. It is observed that the average number of assessed candidate signals $\mathrm{E}$ [ $\left.\mathrm{U}_{\mathrm{ISLM}}\right]$ reduces with increase in iterative threshold and or with increase in $\mathrm{U}$ for ISLM. A reduced number of assessed candidate signals will result in computational complexity Brazilian Microwave and Optoelectronics Society-SBMO received 08 Dec 2016; for review 09 Dec 2016 ; accepted 02 Jun 2017 
reduction or saving in computational resources. We define another metric to measure the saving in computational resources as computational complexity reduction ratio (CCRR).It is defined as

$$
\mathrm{CCRR}=\left[1-\frac{\left.\mathrm{E}_{\mathrm{U}_{\text {ISLM }}}\right]}{\mathrm{U}_{\mathrm{SLM}}}\right] * 100
$$

where $\mathrm{U}_{\mathrm{SLM}}$ is number of candidate signal mappings in conventional SLM. From Equation (6), it is implied that a high CCRR is achieved as average number of assessed candidate signals reduces indicating saving in computational resources.

\section{GOLAY HADAMARD PHASE SEQUENCE SET}

In [11] a random sequence set is discussed and no mention of orthogonality of sequences is done whereas the orthogonal Sylvester Hadamard sequence set of [12] is periodic. Hence, Golay Complementary sequence pairs which have found application in radar, spread spectrum due to their low aperiodic property are being considered for ISLM. But these sequences dont find much application as phase sequence set since various methods to constuct Golay complementary pairs (GCP) in [19] does not assure orgthogonality which is prime necessity in ISLM.

To construct an orthogonal phase sequence set, we apply construction proposed in [20] which is stated as follows:

Let $\mathrm{a}$ and $\mathrm{b}$ be complememtary sequence pair. Let $\mathrm{A}$ and $\mathrm{B}$ be $\mathrm{M} * \mathrm{M}$ circulant matrices constructed with first rows as a and $\mathrm{b}$, respectively. Then we construct a new matrix of order $2 \mathrm{M}$ which is orthogonal as given below.

$$
\left[\begin{array}{cc}
A & B \\
B^{T} & -A^{T}
\end{array}\right] \text { or }\left[\begin{array}{cc}
A & -B \\
B^{T} & A^{T}
\end{array}\right]
$$

Hadamard construction in both cases implies a orthogonal set but GH is based on GCS while H set is based on Walsh functions.

We further analyze above phase sequences of two phase sequence sets for their periodic (PACF), aperiodic autocorrelation function (AACF) and aperiodic cross correlation function (ACCF) for non-zero delay (lag) shift between them to determine which is more aperiodic.

Periodic Autocorrelation Function (PACF) is essentially the autocorrelation of a signal with itself at different points in time, as a function of lag between points of time. 


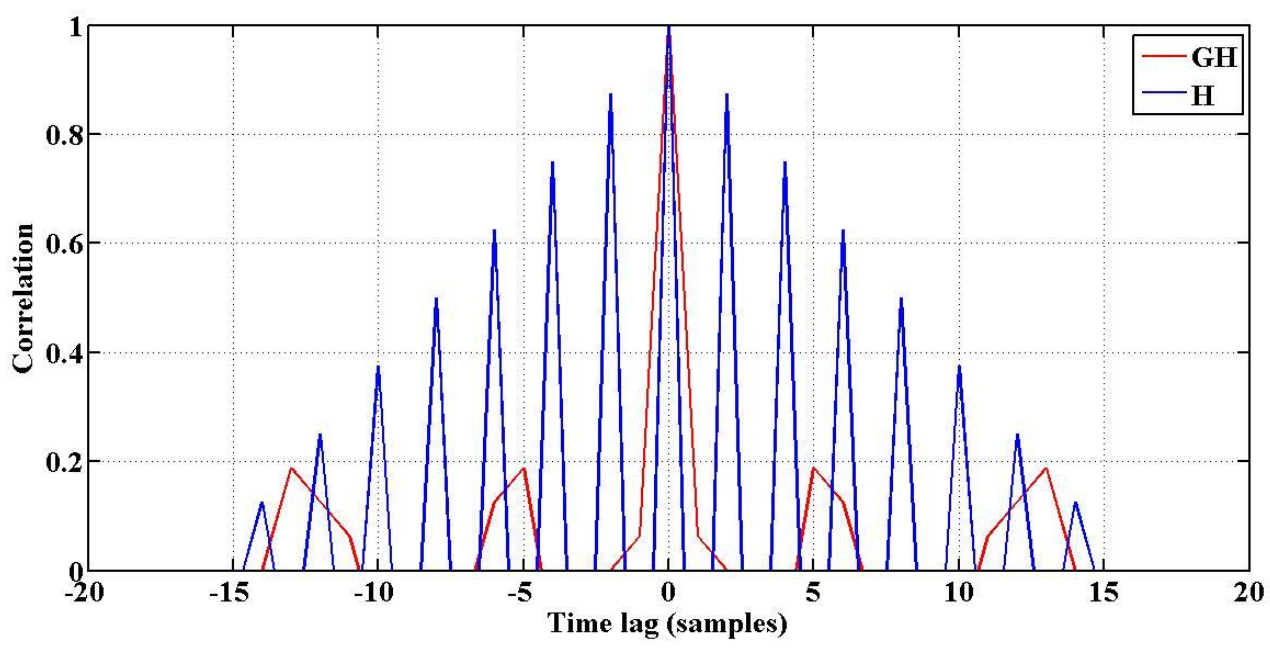

Fig.1a Periodic ACF of Phase sequence sets.

Aperiodic Autocorrelation Correlation function (AACF), is the measure aperiodic behavior of a phase sequence.Consider $\mathrm{a}=\left(\mathrm{a}_{0}, \mathrm{a}_{1}, \ldots . \mathrm{a}_{\mathrm{M}-1}\right)$ be a sequence of length $\mathrm{M}$ such that $\mathrm{a} €(+1,-1)$. Then we define the Aperiodic Auto-Correlation Function (AACF) of a sequence by

$$
\rho_{\mathrm{a}}(\mathrm{k})=\sum_{\mathrm{i}=0}^{\mathrm{M}-\mathrm{k}-1} \mathrm{a}_{\mathrm{i}} \mathrm{a}_{\mathrm{i}+\mathrm{k}}=\mathrm{a}_{0} \mathrm{a}_{\mathrm{k}}+\mathrm{a}_{1} \mathrm{a}_{\mathrm{k}+1}+\mathrm{a}_{2} \mathrm{a}_{\mathrm{k}+2}+\ldots \ldots . \quad 0 \leq \mathrm{k} \leq \mathrm{M}-1
$$

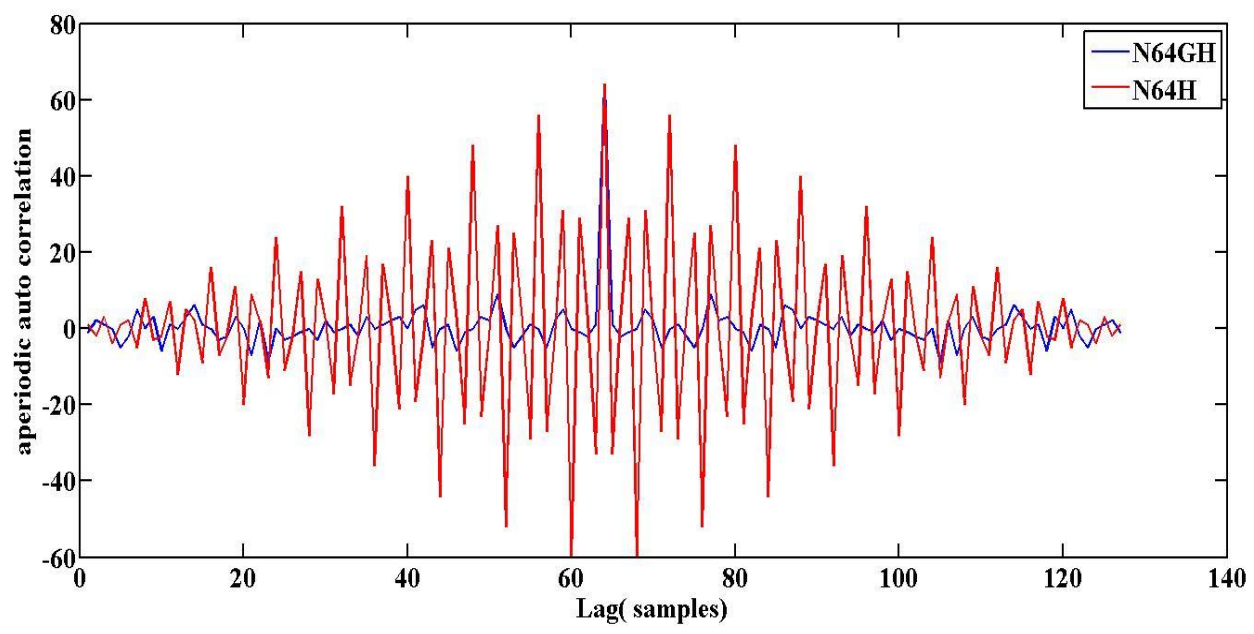

Fig.1b AACF of Phase sequence sets.

Similarly, Aperiodic cross correlation (ACCF) is the correlation between two sequences $(a, b)$. We validate through simulations shown in Fig.1a, Fig.1b and Fig.1c that GH sequences exhibit improved periodic and aperiodic correlation properties compared to $\mathrm{H}$ sequences.

The merit factor of a sequence is a measure of quality of sequence. It is defined for a sequence a of length $\mathrm{M}$ as 


$$
\mathrm{MF}=\frac{\mathrm{M}^{2}}{2 \sum_{\mathrm{k}=1}^{\mathrm{M}-1}\left|\rho_{\mathrm{a}}(\mathrm{k})\right|^{2}}
$$

ACF and AACF characterize the behavior of individual sequences and not the performance of the entire phase sequence set. To analyze performance of the entire phase sequence set, the average mean-square value of auto correlation $\mathrm{R}_{\mathrm{AC}}$ and maximum value of aperiodic cross correlation function $\left(\mathrm{C}_{\mathrm{MAX}}\right)$ are considered. The auto correlation measure $\mathrm{R}_{\mathrm{AC}}$ is given by

$$
\mathrm{R}_{\mathrm{AC}}=\frac{1}{\mathrm{P}} \sum_{\mathrm{i}=1 \mathrm{k}=1-\mathrm{M}, \mathrm{k} \neq 0}^{\mathrm{M}-1}\left|\rho_{\mathrm{i}, \mathrm{i}}(\mathrm{k})\right|^{2}
$$

where $\mathrm{P}$ is number of sequences in the set. In our case, $\mathrm{P}=\mathrm{M}$ since a square phase sequence set is generated.



Fig.1c ACCF of Phase sequence sets.

Reduction in $\mathrm{R}_{\mathrm{AC}}$ indicates weak correlation among sequences in a set and therefore low PAPR is realizable in ISLM. Lower peaks in ACCF or higher value of merit factor (MF) implies more aperiodic sequence while reduced $\mathrm{C}_{\mathrm{MAX}}$ results in lower bit error rate.

We consider low $\mathrm{C}_{\mathrm{MAX}}$, low $\mathrm{R}_{\mathrm{AC}}$ and low $\mathrm{AACF}$ (or high MF) as optimization criteria in selection of phase sequence sets and evaluate the PAPR performance and complexity of ISLM. From Table 1, it is inferred that the new sets of orthogonal sequences are characterized with lesser peaks in aperiodic cross-correlation functions, and exhibit good autocorrelation properties too. The improved correlation properties along with the design of the set satisfying above two necessary criteria proves that GH sequence set will achieve improved PAPR reduction for ISLM-OFDM. 
TABLE I: CORRELATION PROPERTIES OF PHASE SEQUENCE SETS

\begin{tabular}{|c|c|c|}
\hline Parameter & GH set & H set \\
\hline & \multicolumn{2}{|c|}{ 64-bit sequence set } \\
\hline $\mathrm{C}_{\mathrm{MAX}}$ & 0.9843 & 0.9843 \\
\hline $\mathrm{R}_{\mathrm{AC}}$ & 0.74 & 5.74 \\
\hline $\mathrm{MF}$ & 0.4050 & 0.0982 \\
\hline
\end{tabular}

\section{RESULTS AND DISCUSSION}

The PAPR and complexity performances of conventional SLM and proposed method is investigated by computer simulations. The proposed SLM has N=64 subcarriers, 1/2 rate Convolutional Encoding and 16 QAM mapping. The performance evaluation considers Hadamard (H) and Golay Hadamard (GH) phase sequence sets for ISLM while Hadamard phase sequence set is considered for conventional SLM. The iterative threshold is varied between $4 \mathrm{~dB}$ to $8 \mathrm{~dB}$ and $\mathrm{U}=4,8,16$ and 32 .

In Fig. 2 we compare the CCDF of GH and $\mathrm{H}$ sequence set for ISLM for iterative threshold of $6 \mathrm{~dB}$. It is seen that for a given value of $\mathrm{U}, \mathrm{GH}$ sequence set exhibits improved PAPR reduction compared to Hadamard sequence set. This is because GH sequence set is more aperiodic compared to Hadamard sequences as discussed in section III.

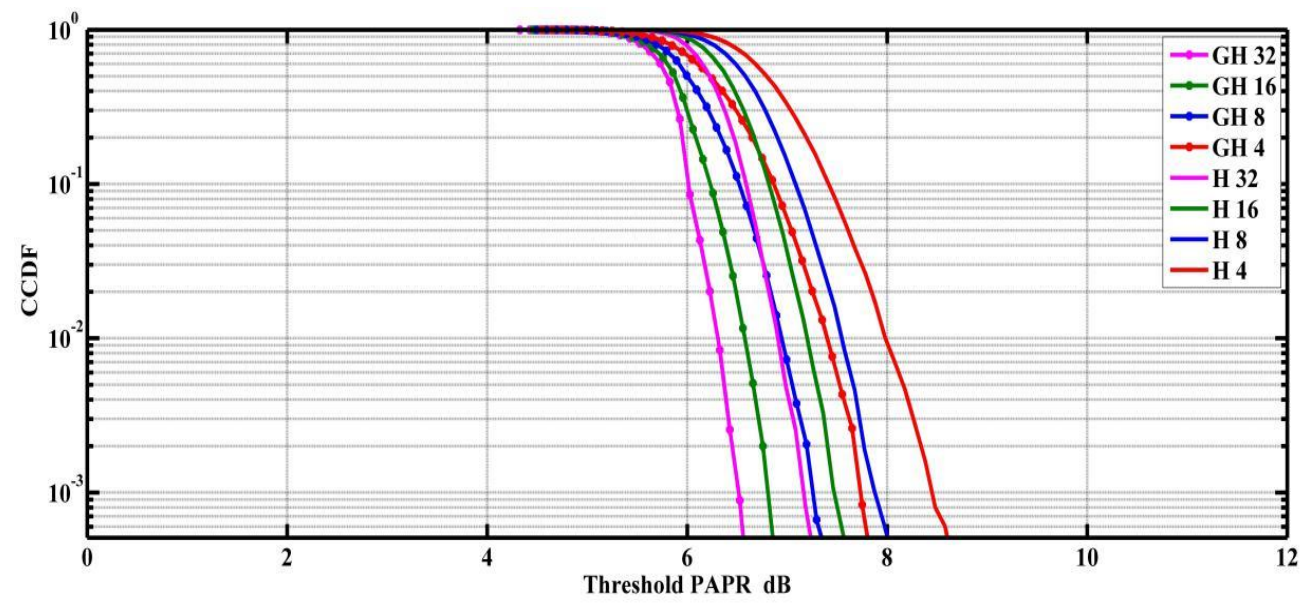

Fig.2. PAPR Analysis for different Orthogonal Phase sequence sets for ISLM

Fig.3 shows the CCDF of PAPR for various values of $U$ to compare PAPR improvement of ISLM with GH phase sequence set over conventional SLM. It is obvious that ISLM offers significant gain in PAPR compared to conventional SLM and OFDM (no PAPR reduction). A PAPR gain of about $3.7 \mathrm{~dB}$ Brazilian Microwave and Optoelectronics Society-SBMO received 08 Dec 2016; for review 09 Dec 2016; accepted 02 Jun 2017 
is achieved compared to OFDM for 32 candidate signals at iterative thresholdof $6 \mathrm{~dB}$. PAPR gain improves as $\mathrm{U}$ increases. ISLM with $\mathrm{U}=4$ gives same performance as conventional SLM with $\mathrm{U}=16$. Also, ISLM with $U=8$ has identical PAPR performance to conventional SLM with $U=32$. This is a computational saving since ISLM utilizes lesser number of candidate signals for same PAPR performance.

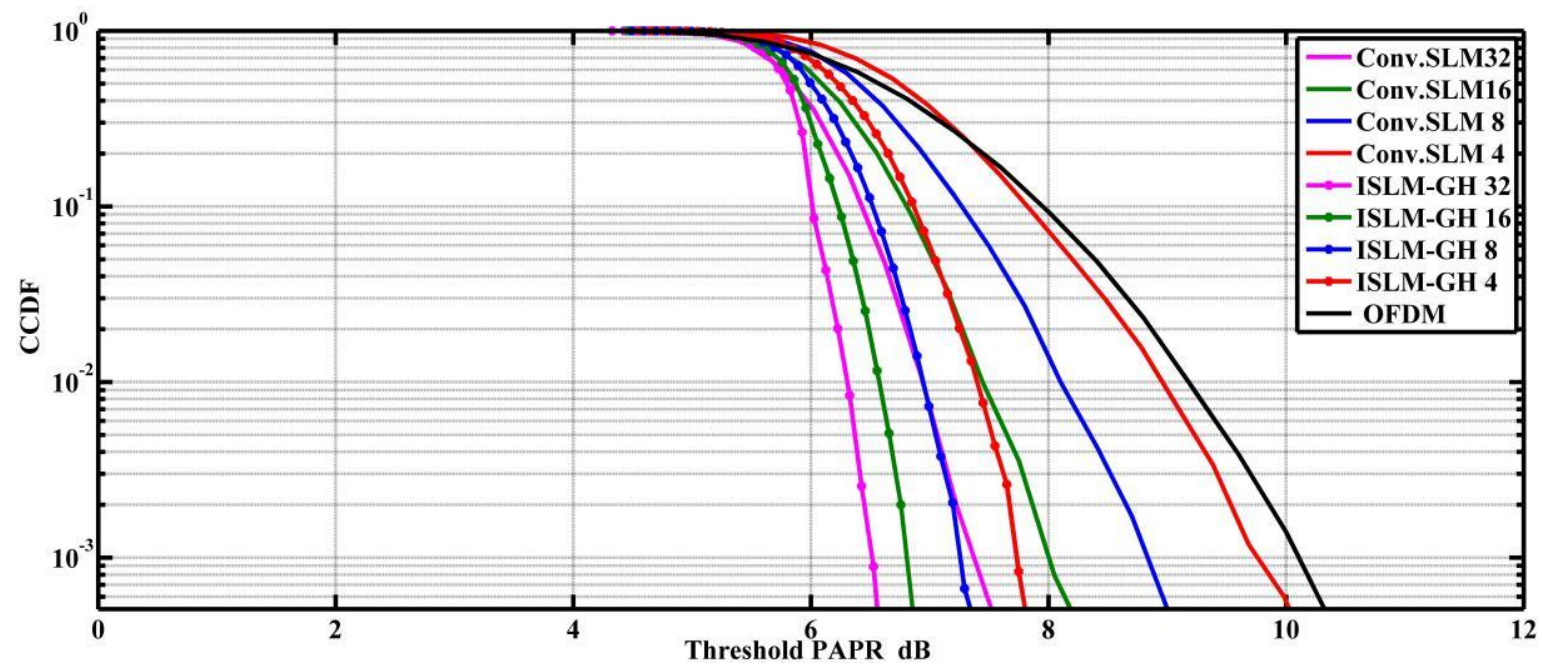

Fig.3. CCDF of Iterative SLM with GH sequence set

Table II compares achieved PAPR for different values of $\mathrm{U}$ at CCDF of $10^{-3}$ for Conventional SLM and ISLM with GH and $\mathrm{H}$ phase sequence sets. The proposed method outperforms conventional SLM but still falls short of the theoretical achievable PAPR of SLM. At low values of $U$, the improvement in PAPR reduction is more compared to conventional SLM.

The average number of assessed candidate signals computed based on (5) is as shown in Table III. It decreases as a function of threshold PAPR while for Conventional SLM (Conv. SLM), the average number of assessed candidate signals remains equal to value of U. Especially at threshold values above $4 \mathrm{~dB}$, the assessed number of candidate signals is independent of $U$ and computational complexity approaches that of OFDM.

The simulation results indicate $\mathrm{E}\left[\mathrm{U}_{\mathrm{SLM}}\right]$ decreases as iterative threshold increases satisfying (5). For $\mathrm{U}$, less than or equal to 16 , for iterative thresholds up to $5 \mathrm{~dB}$ number of assessed candidate signals is same as conventional SLM. But beyond $5 \mathrm{~dB}$ it decreases as iterative threshold increases and is close to $1 \mathrm{~dB}$ for $8 \mathrm{~dB}$ iterative threshold. We infer that suitable choice of iterative threshold would be $6 \mathrm{~dB}$ or more depending on complexity reduction expected.

Fig.4 presents the average number of assessed candidate signals as a function of iterative threshold. The theoretical values tabulated in Table III indicate a threshold of $5 \mathrm{~dB}$ or more brings about more than 
$80 \%$ reduction of E [ $\left.\mathrm{U}_{\mathrm{SLM}}\right]$ while from Fig.4, it is evident that about $50 \%$ reduction in E [U $\left.\mathrm{U}_{\text {SLM }}\right]$ is achieved.

TABLE II. COMPARISON OF THRESHOLD PAPR ACHIEVABLE AT CCDF OF $10^{-3}$

\begin{tabular}{|c|c|c|c|c|}
\hline Method & $\mathbf{U = 4}$ & $\mathbf{U = 8}$ & $\mathbf{U = 1 6}$ & $\mathbf{U = 3 2}$ \\
\hline Analytical Result (4) & $6.4 \mathrm{~dB}$ & $5.1 \mathrm{~dB}$ & $4.3 \mathrm{~dB}$ & $3.8 \mathrm{~dB}$ \\
\hline ISLM-GH & $7.8 \mathrm{~dB}$ & $7.3 \mathrm{~dB}$ & $6.8 \mathrm{~dB}$ & $6.5 \mathrm{~dB}$ \\
\hline ISLM-H & $8.5 \mathrm{~dB}$ & $7.9 \mathrm{~dB}$ & $7.4 \mathrm{~dB}$ & $7.2 \mathrm{~dB}$ \\
\hline Conv. SLM & $9.8 \mathrm{~dB}$ & $8.8 \mathrm{~dB}$ & $8 \mathrm{~dB}$ & $7.5 \mathrm{~dB}$ \\
\hline
\end{tabular}

Fig.5 shows the graph of CCRR achieved for GH and $\mathrm{H}$ phase sequence sets. As number of assessed candidate signals decreases, CCRR increases satisfying (6). It is observed that for values of U larger than 16, higher saving in computational resources occurs. There is $65 \%$ and $50 \%$ reduction in computational complexity for 32 candidate signals for $\mathrm{GH}$ an $\mathrm{H}$ phase sequence set respectively. For values of $U$ greater than 32 , there is only a marginal improvement in CCRR. Interestingly, it is evident that complexity reduction is higher for a $\mathrm{GH}$ sequence set compared to $\mathrm{H}$ sequence set. This is obvious considering the analysis presented in section III.

TABLE III THEORETICAL E [ U SLM ] FOR ISLM

\begin{tabular}{|c|c|c|c|c|c|c|}
\hline \multirow{2}{*}{$\mathbf{U}$} & \multicolumn{6}{|c|}{ Threshold PAPR dB } \\
\cline { 2 - 7 } & $\mathbf{2}$ & $\mathbf{3}$ & $\mathbf{4}$ & $\mathbf{5}$ & $\mathbf{6}$ & $\mathbf{7}$ \\
\hline 8 & 7.99 & 7.01 & 3.08 & 1.54 & 1.17 & 1.06 \\
\hline 16 & 15.91 & 12.15 & 3.25 & 1.54 & 1.17 & 1.06 \\
\hline 32 & 31.95 & 18.68 & 3.26 & 1.54 & 1.17 & 1.06 \\
\hline
\end{tabular}

\section{CONCLUSIONS}

In this paper, investigation of PAPR and complexity performance of low complexity SLM for different orthogonal phase sequence sets has been performed. A detailed analysis of the various parameters which influence the choice of phase sequence set is carried out. A phase sequence set with low AACF for ISLM is more suitable choice since it achieves both PAPR reduction and reduced complexity. Since orthgonal sequences are used, side information need not be transmitted. Therfore bit loading is avoided in our approach compared to [11]. As the number of assessed candidate signals per 
OFDM sample is variable,the number of complex additions and multiplications performed in the IFFT section is also reduced. It is concluded that the choice of phase sequence set, iterative threshold and the maximum number candidate signal mappings influence the reduction in computational complexity in a N sub-carrier iterative SLM system.

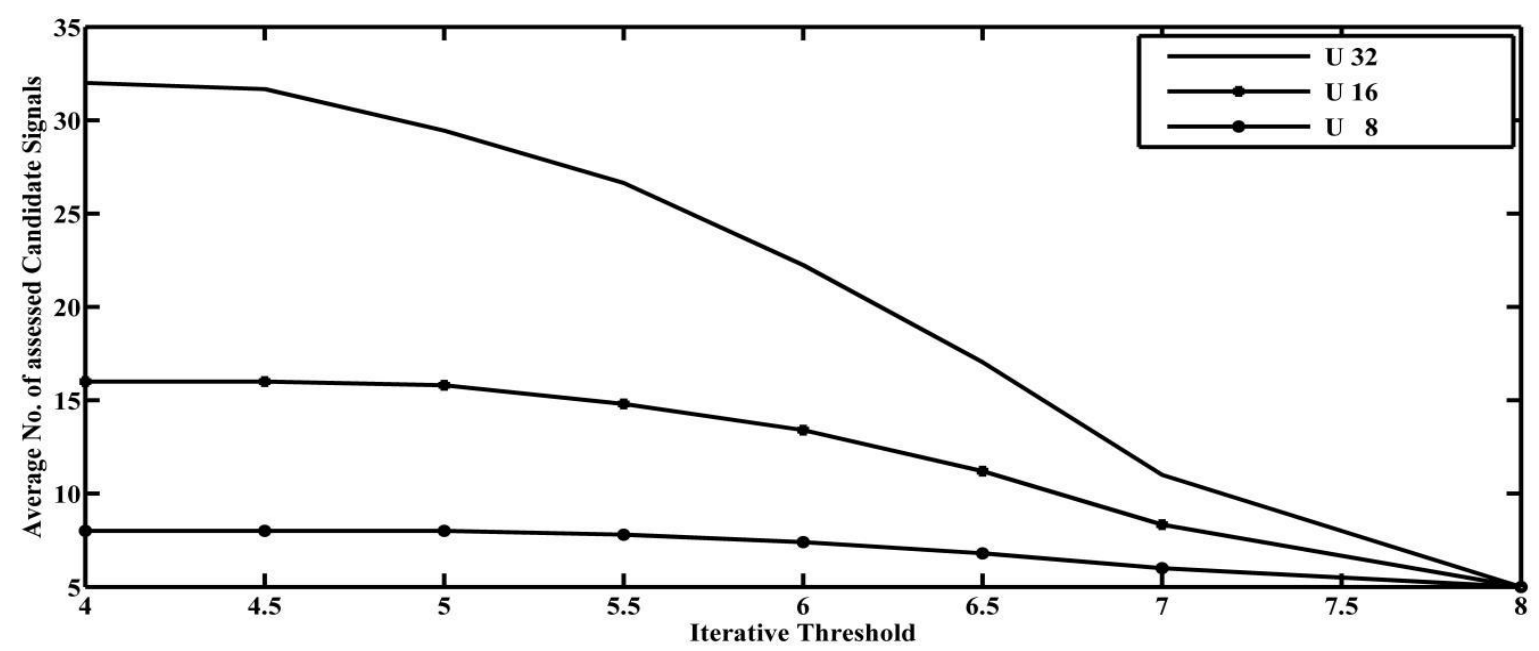

Fig. 4 Number of assessed candidate Signals Versus Iterative Threshold

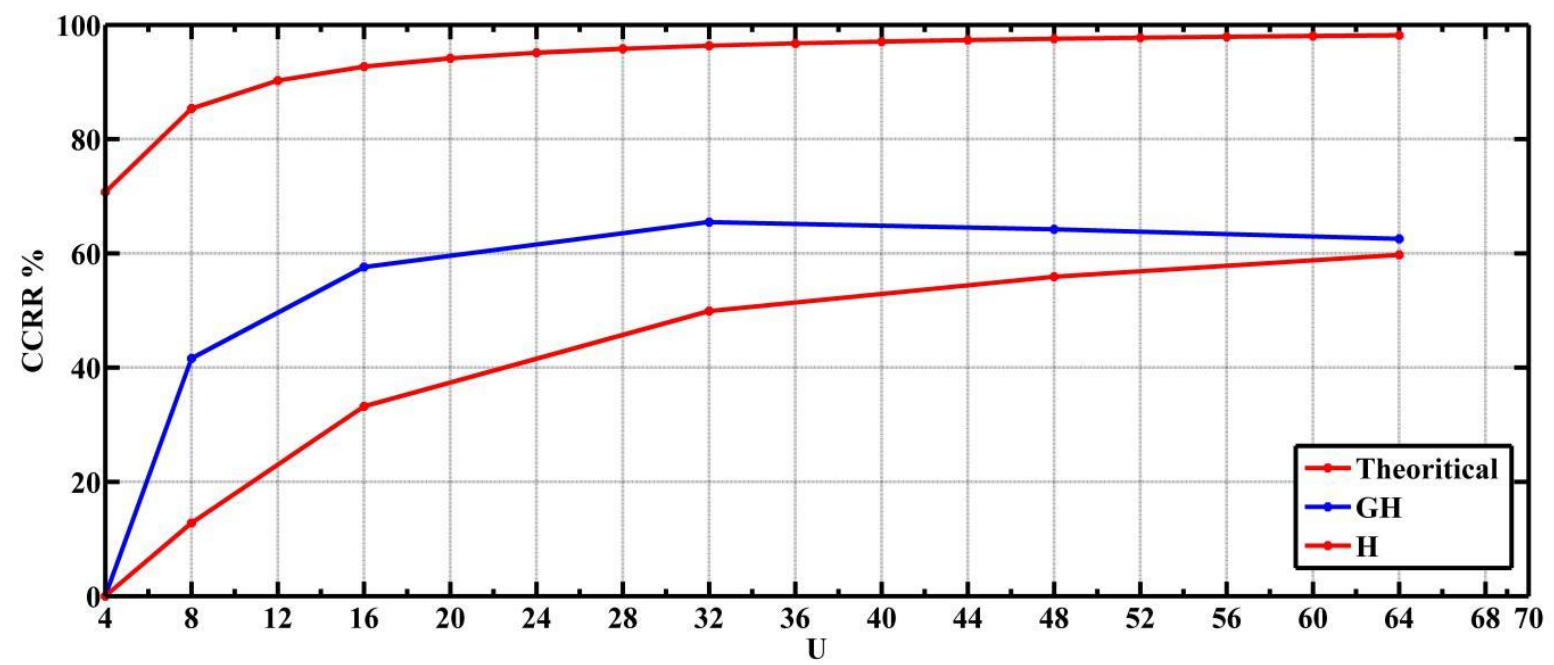

Fig.5. Complexity Analysis of different phase sequence sets

\section{REFERENCES}

[1] Bauml, R., Fischer, R., and Huber, J., "Reducing the peak-to-average power ratio of multicarrier modulation by selected mapping," IEE Electronics Letters, vol. 32, pp. 2056-2057, Oct. 1996.

[2] Park, Sung-Ho, Jong-Yeop Lee, Eon-Pyo Hong, Seung-Wook Min, and Dong-Soo Har, "Merged SLM scheme for PAPR reduction of OFDM system with low complexity," SARNOFF,2009,pp.1-5.

[3] Rahman, M.S. Rahim, N.A.S. Bhuiyan and S.Ahmed,“ PAPR reduction of OFDM system using condexmatrix basedSLM method with low computationaloverhead," in 2nd International Conference on Electrical Information and Communication Technology (EICT),Khulna, 2015,pp.294-297.

Brazilian Microwave and Optoelectronics Society-SBMO received 08 Dec 2016; for review 09 Dec 2016; accepted 02 Jun 2017 
Journal of Microwaves, Optoelectronics and Electromagnetic Applications, Vol. 16, No. 3, September 2017626

DOI http://dx.doi.org/10.1590/2179-10742017v16i3819

[4] Wang, Chin-Liang, andYuan Ouyang,"Low complexity selected mapping schemes for peak-to-average power ratio reductionin OFDM systems,"IEEE Transactions on Signal Processing,vol.53,pp.4652-4660,2005.

[5] Ji, Jinwei, Guangliang, Ren, and Huining Zhang, "A semi-blind SLM scheme for PAPR reduction in OFDM systems with low-complexity transceiver," IEEE Transactions on Vehicular Technology,Vol. 64 no.6,pp.2698-2703,2015.

[6] Yang, Suckchel and Yoan Shin,"Partitioned SLM scheme with low complexity for PAPR reduction of OFDM signals,"in IEEE $17^{\text {th }}$ International Symposium in Personal,Indoor and Mobile Radio Communication,2006,pp.1-5.

[7] Jeon, Hyun-Bae,Jong-Seon No, and Dong-Joon Shin,“A low complexity SLM scheme using additive mapping sequences for PAPR reduction of OFDM signals,"IEEETransactions on Broadcasting,vol.57,no.4,pp.866-875,2011.

[8] K.. Kim,H. B. Jeon, J. S. No and D. J. Shin, "Low-complexity selected mappingscheme usingcyclic-shifted inverse fast Fourier transform for peak-to-average power ratio reduction in orthogonal frequency division multiplexing systems," in IET Communications, vol.7 no.8, pp.774-782, May 2013.

[9] L.Yang, Y.M. Siu, K.K. Soo, S.W.Leung, S.Q. Li, "Low-complexity PAPR reduction technique for OFDM systems using modified widely linear SLM scheme," AEU-International Journal of Electronics and Communications, Vol.66, no.12, pp.1006-1010, December 2012.

[10] Baxley,R.J.,\&Zhou,G.T.,“Assessingpeak-to-average power ratios for communicationsapplications,”Military Communications Conference, MILCOM ,Vol. 1,2004, pp. 181-185.

[11] Siegl,C.,\&Fischer,R. F., “Asymptotic performance analysis and successive selected mapping for PAR reduction in OFDM," inIEEETransactions on Signal Processing, vol.58,no.6,pp. 3228-3237,2010 .

[12] Dae-Woon Lim, Seok-Joong Heo, Jong-Seon No, and Habong Chung, "On the phase sequence set of SLM OFDM scheme for a crest factor reduction,” IEEE Transactions on Signal Processing, vol.54,no.5,pp.1931-1935,Oct 2006.

[13] Meymanatabadi, Saber, Javad Musevi Niya, and Behzad Mozaffari,"Selected mapping technique for PAPR reduction without side information based on m-sequence,"Wireless Personal Communications,vol.71,no.4,pp.25232534,2013

[14] Adegbite, Saheed A.,Scott McMeekin, and Brian G.Stewart,"Modified shapiro-rudin sequences for SLM-PAPR reduction in wireless OFDM systems,"9th International Symposium on Communication Systems, Networks \& Digital Signal Processing (CSNDSP), 2014 . DOI: 10.1109/CSNDSP.2014.6923844 .

[15] Chandwani, Mohit, et al.,"A low complexity SLM technique for PAPR reduction in OFDM using Riemann sequence and thresholding of power amplifier,"Annual IEEE India Conference2009.

[16] Wysocki BJ \& Wysocki TA, "Optimization of orthogonal polyphase spreading sequences for wireless data applications," IEEE VTS 54th Vehicular Technology Conference, 7-11 October 2001, 3, 1894-1898. Copyright IEEE 2001.

[17] Z.Zhang, F.Tian, F.Zeng ,L.Ge and G.Xuan,"Mutually orthogonal complementary pairs for OFDM-CDMA systems," 12th International Conference on Signal Processing (ICSP), Hangzhou, pp. 1761-1765,2014.

[18] Liu, Zilong, Yong Liang Guan, and Udaya Parampalli,"New complete complementary codes for peak to mean power control in multi-carrier CDMA," IEEE Transactions on Communications, Vol.62,no.3,1105-1113,2014. 
[19] F.Fiedler, J.Jedwab and M.G.Parker,“A framework for the construction of Golay sequences,”IEEETrans.Inf.Theory, vol. 54, no.7, pp. 3114-3129, June 2008.

[20] Seberry, Jennifer R., Beata J. Wysocki, and Tadeusz A. Wysocki, "On a use of Golay sequences for asynchronous DS CDMA applications,” Advanced Signal Processing for Communication Systems, Springer US, pp.183-196,2002. 